\title{
Boris DJUBO
}

\section{Bilderlosigkeit und anthropomorphe Darstellungen in den deutschen und niederländischen Grammatiken des Barock: sprachwissenschaftlich verursachte und konfessionell motivierte Sichtweise}

\begin{abstract}
Human figures were a suitable means of assigning human qualities to non-human entities, thereby making an abstract phenomenon more concrete and easier to understand. During the baroque period, one of the widely used techniques in the works of German and Dutch grammarians was visual presentation of their material in tables. Illustrative examples or tables and dichotomies were differently preferred as visual material in the language teachings of representatives of the confessions as well as the most important philosophical and grammatical theories, which were respected and had authority in the $17^{\text {th }}$ century. The iconoclasm of the Calvinist authors, that is, aniconism and removal of images from churches, correlated with the reluctance of the grammarians to visualise their subjects in pictures. For presenting the contents, language teachers chose a frugal form, namely the tables and the dichotomic method, which is usually manifest in the composition of grammar books and description of the most important characteristics of the parts of speech.
\end{abstract}

Keywords: confessional stratification, early modern period, Protestantism, Calvinism, grammaticography, visual design, iconoclasm.

\section{Einleitung}

In dieser Abhandlung soll dargelegt werden, inwieweit die Verwendung von Bildern, die den menschlichen Körper darstellen, von der grammatik-theoretischen und religiös-philosophischen Position der jeweiligen Verfasser von Werken über die Grammatik abhängig ist. 
Es werden hier die wichtigsten Sprachlehren besprochen, die den Grammatiken des Deutschen und des Niederländischen gewidmet sind, wie auch lateinische Grammatiken deutscher und niederländischer Autoren. Die Betrachtung der deutschen Grammatiken beginnt mit den ersten Sprachlehren in der deutschen Sprache: den Werken von Peter Jordan (1533) und Jacob Grüßbeutel (1534). Sie wird bis zur Grammatik von Tilemann ([Olearius] 1630) fortgesetzt. Unter den ersten niederländischen Grammatiken werden hier die bekanntesten vorgestellt: Twe-spraack vande nederduitsche letterkunst von Hendrik Laurenszoon Spieghel (1584) und De Nederduytsche Grammatica von Christiaen van Heule (1625). Die letztere ist laut $\mathrm{H}$. Glück die wichtigste Grammatik des Niederländischen des 17. Jh. und stellt einen der Anfänge der niederländischen Grammatikographie dar (Glück 2002: 321-322).

Mit der Auswahl der in Deutschland und in den Niederlanden erschienenen Grammatiken aus der ersten Hälfte des 17. Jahrhunderts (aus dem Frühbarock) und teilweise aus dem 16. Jahrhundert (aus der vorbarocken Zeit) wird ein Zeitraum festgelegt und als Epoche der Grammatikographie ausgewiesen, in der die Welle des reformatorischen Bildersturms und seine Auswirkungen besonders deutlich zu spüren waren. In dieser Zeit wurde unter den deutschen und niederländischen Sprachlehrern auch eine besonders strenge Spaltung in der Bilderfrage beobachtet.

Bilder und Schemata, die für die Realisierung einer kommunikativen Absicht eingesetzt werden, werden in dieser Abhandlung zu den außersprachlichen Komponenten gezählt. Nach der mit dem Entstehen der Semiotik aufgekommenen Auffassung

bezeichnet der Ausdruck Bild Gegenstände, die als spezielle Zeichen innerhalb einer Mitteilungs- oder Ausdruckshandlung (im Unterschied zur sprachlichen Darstellung) nicht zur Beschreibung, sondern zur visuellen Veranschaulichung bzw. Klassifikation eines (fiktiven oder realen) Sachverhalts verwendet werden (Hombach 2002: 6).

Unter den Menschenbildern in den Grammatiken des Barock seien zwei Darstellungsarten unterschieden: einerseits anthropomorphe ikonographische Darstellungen der Grammatik oder ihrer Begriffe und andererseits natürliche Gegenstände (in unserem Fall menschliche Wesen).

\section{Anthropomorphe ikonographische Darstellungen der Grammatik und ihrer Begriffe}

Schon die mittelalterlichen Holzschnitte setzten zusätzlich zur verbalen Verkündigung heiliger Texte auf Visualisierungen zur Verbreitung des religiösen Heilswissens, die sich vor allem an den nicht-literaten Teil der Bevölkerung wandten 
(Schnettler \& Pötzsch 2007: 473). Diese Technik spielt eine wichtige Rolle besonders im Anfangsunterricht der Grammatik. Um den Erkenntnisprozess zu stimulieren, soll man die Sinne der Schüler so weit wie möglich aktivieren: das betrifft das Hören, das Sehen und den Tastsinn.

Menschliche Figuren waren ein durchaus geeignetes Mittel, um die menschlichen Qualitäten den nicht-menschlichen Entitäten zuzuordnen und das abstrakte Phänomen dadurch konkreter und leichter verständlich zu machen. Grammatik wurde von personifizierten Frauen mit verschiedenen Kombinationen von Eigenschaften ausgestattet.

Abbildung 1 zeigt die Grammatik in Form einer Skulptur auf dem Königsportal der Kathedrale Notre Dame von Chartres. Die Frau hält eine Peitsche und ermahnt die Schüler, fleißig zu lernen (vgl. Mittelberg 2002: 77).

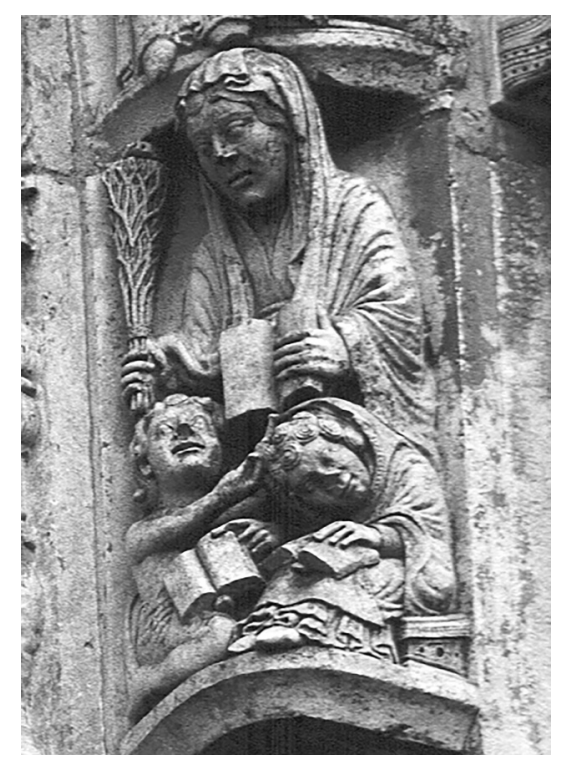

Abbildung 1. Grammatik in der Kathedrale Notre Dame von Chartres; Quelle: Mittelberg 2002: 77

Hier ist das konventionale Sujet - die Allegorie - vorhanden. Mit der Inhaltsdeutung in der bildenden Kunst beschäftigt sich die Ikonografie. Wie Erwin Panofsky schreibt:

Die Identifizierung solcher Bilder, Anekdoten und Allegorien ist der Bereich dessen, was normalerweise mit der Bezeichnung 'Ikonographie' gemeint ist. In der Tat meinen wir, wenn wir zwanglos vom 'Sujet im Gegensatz zur Form' sprechen, hauptsächlich den Bereich des sekundären oder konventionalen Sujets, nämlich die Welt spezifischer, sich in Bildern, Anekdoten und Allegorien manifestierender Themen oder Konzepte im Gegensatz zum Bereich des primären oder natürlichen Sujets, wie es sich in künstlerischen Motiven manifestiert (Panofsky 1975: 39). 
Metaphern spielen beim Lernen (Wissensbildung) eine wichtige Rolle. Durch strukturelle, visuelle und funktionale Analogien können neue Inhalte wesentlich schneller erfasst und verstanden werden. Die Ergebnisse der Untersuchungen auf dem Gebiet der Metapher und der Metonymie zeigen,

dass Raumkonzepte (Gebäude, Behälter, Raumstrukturen im Allgemeinen), der Mensch (im Hinblick auf seine physische und soziale Eigenschaften) und soziale Hierarchien (feudale- und Sozialsysteme) bedeutende Quelldomänen für die metaphorische und metonymische Strukturierung des Konzepts ‘Grammatik’ sind (Mittelberg 2002: 85).

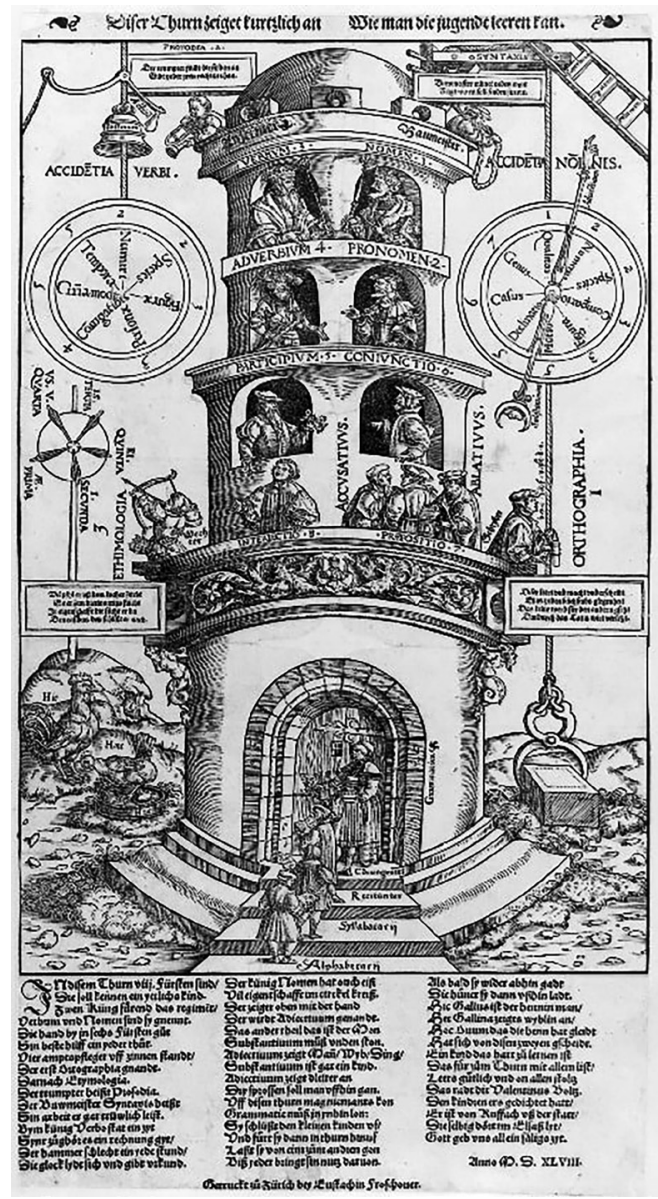

Abbildung 2. Heinrich Vogtherr der Ältere, Turm der Grammatik, 1548; Quelle: Vogtherr 1548, No. 1430, https://commons.wikimedia.org/wiki/File:Vogtherr_Turm_der_Grammatik.jpg

Der menschliche Körper dient als primäre Basis für die meisten abstrakten Konzepte und somit für eine ganze Reihe von komplexen Metaphern (Firth 1990: 
242 ff.). Nicht ohne Grund spricht man in diesem Zusammenhang von "der Verkörperung des Geistes" (the embodiment of mind) (Lakoff \& Johnson 1999: 16 ff., zit. nach Nasalski 2004: 128).

Im 16. Jahrhundert wurde der Grammatica ihr eigenes Gebäude, der Turm der Grammatik, zugeordnet.

In einem um 1548 in Zürich erschienenen Einblattdruck der Humanisten Heinrich Vogtherr d. Ä. (Holzschnitt) und Valentin Bolz (Text) ist die Grammatik die Bewohnerin des gesamten Turms.

Das Thema 'Sprache' und das Motiv 'Turm' besitzen eine lange gemeinsame Tradition. Zahlreich sind vor allem die sich auf die Bibel stützenden Bilder des Turms von Babel. Die Tradition der Architekturmetaphorik im sprachtheoretischen Diskurs wird in den sprachtheoretischen Schriften von Justus Georg Schottelius einprägsam greifbar. Schottelius bezeichnet die deutsche Sprache und ihre Grammatik als "Kunstgebäu" (Schottelius 1663: 50). Die Personifizierungen fehlen auch bei Schottelius nicht, z.B. die Dialekte und sprachverwandte Gruppen:

Um die Säulen herum stehen soldatisch gekleidete männliche Gestalten mit nach oben gewandtem Blick. Durch ihre Kleidung zeigen sie ihre unterschiedlichen Herkunftsregionen an, es sind die Repräsentanten der verschiedenen Mundarten und sprachverwandten Völker, die alle gemeinsam auf das sie verbindende und als virtuelles System über ihnen stehende Hochdeutsche blicken (Moulin 2011: 34).

\title{
3. Darstellungen natürlicher Gegenstände (menschlicher Wesen)
}

$\mathrm{Zu}$ visuellen Darstellungen in Grammatikbüchern schreibt Claudine Moulin folgendes:

\begin{abstract}
Neben der bildlichen Gestaltung von Titelblättern begegnet auch der Einsatz von visuellen Elementen innerhalb des Textinnern und mit Bezug zu diesem. Die Illustrierung durch in den Text eingegebene Bilder ist aus der Handschriftentradition des Mittelalters und ihrer Illuminierung bekannt; in der Fachprosa dient sie im Kontext der Wissensvermittlung vor allem erläuternden bzw. didaktischen Zwecken. Das Bild, das visuell abgespeichert wird, stützt somit die memoria und ars docendi des Textinhaltes (Moulin 2008: 20).
\end{abstract}

Im Gegensatz zu den bisher angeführten Bildern gibt es im Weiteren keine Personifikation. Der Alltag wird hier durch körperbezogene Bildschemata abgebildet. Schon in den ersten deutschen Grammatiklehrbüchern gibt es solche Bilder. In Peter Jordans Leyenschul (1533) ist ein Kopf mit einer Narrenkappe dargestellt. Jordan benutzt Bilder, die an den ersten Laut, beziehungsweise an den ersten Buchstaben des betreffenden Wortes erinnern sollten (lewe -1 , messer $-\mathrm{m}$, nar - n, usw.).

Jacob Grüßbeutel stellt in seinem Stimen büchlein mit figuren (1534) die Assoziationen zwischen Buchstaben und ihren Formen mit Lauten von Tier und 
Mensch dar. So sehen wir eine Frau, die Hühner vor sich hertreibt. Bei diesem Vorgang kommt der Laut als Naturlaut vor (vgl. Müller 1882: 110 ff.; Fijałkowski 2008: 462).

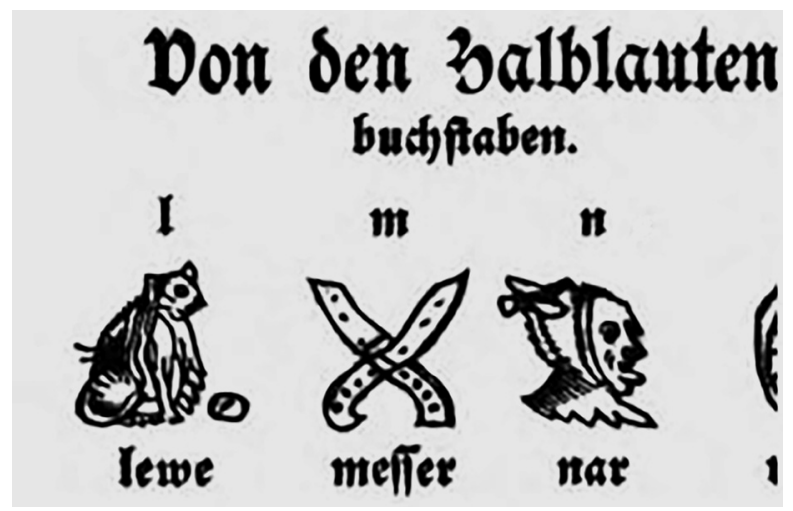

Abbildung 3. Von den halblauten Buchstaben aus Jordan, 1533; Quelle: Jordan 1882: Aiiiij

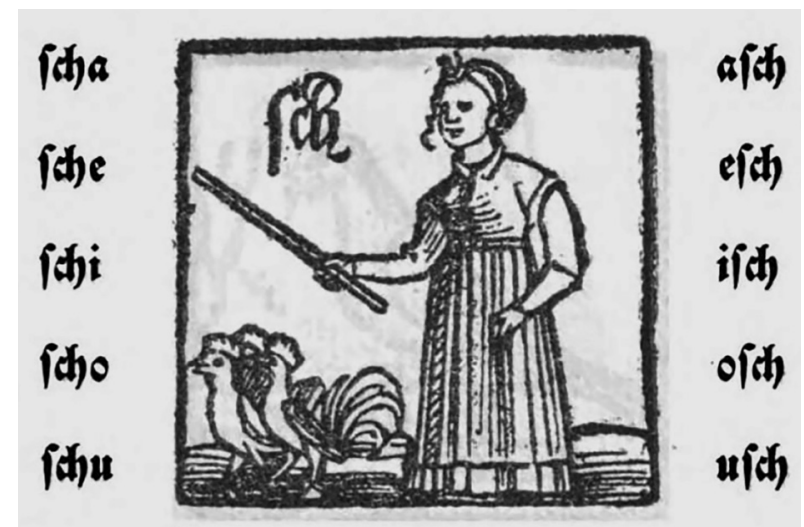

Abbildung 4. Eine Frau, die Hühner vor sich hertreibt (1534); Quelle: Grüßbeutel 1882, n.p.

Die dem Text beigefügten und visuell angereicherten Hilfsmittel der Wissensvermittlung wurden z.B. von Tilemann Olearius (1600-1671) in seiner 1630 erschienenen Deutschen Sprachkunst ([Olearius] 1630) realisiert, die letztlich ein Leitfaden für den Elementarunterricht ist. Robert Hanns bemerkt über Olearius' Grammatik:

das werkchen macht, soweit wir wissen, zum ersten male den versuch, die psychologische begründung seiner lehrart zu geben, und bietet dann einen 'modus docendi durch vnterschiedene bilder' (Hanns 1881: 31-32). 
Olearius schreibt bei der Behandlung der Zeitformen:

Weil die lehre von den temporibus in doctrina verborum, meines erachtens/ die schwerste ist/ vnd von den kleinen Kindern nicht so bald gefasset/ habe ich die selbe in folgende Bildnisse vnnd imagines Mnemonicas bringen wollen/ welche dann den Kindern ganz leicht mögen eingebildet werden ([Olearius] 1630: 58).

In der Inszenierung liefert die Geste eine wichtige Information über das Geschehen und die darin befindlichen Personen. Die Gebärdensprache gilt als der genauere Ausdruck des inneren Lebens eines Menschen als die Worte, die im größeren Maße durch das Bewusstsein gesteuert werden. Inhaltlich kann die Geste das sprachliche Zeichen wiederholen, es verstärken, hervorheben oder betonen.

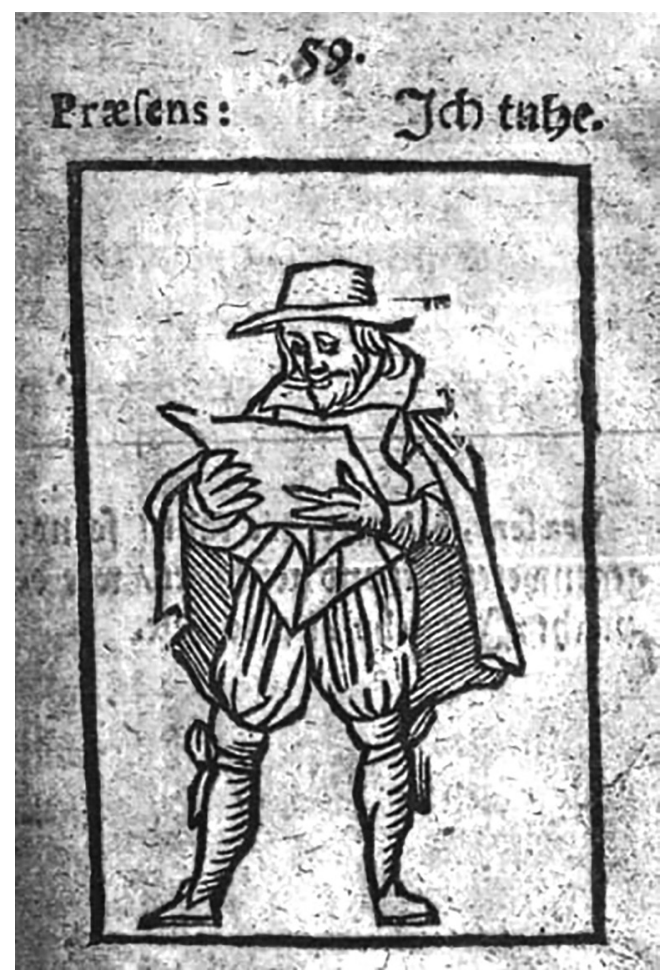

Abbildung 5. Der Praesens (1630); Quelle: [Olearius] 1630: 59

"Praesens, bedeutet die taht, so noch gegenwärtig ist/ vnd geschihet/ wie der spricht: Ich steh/ oder ich lese" ([Olearius] 1630: 60). Der Augenblick wird bei der Darstellung der Gegenwart festgehalten. Da sehen wir einen breitbeinig stehenden Mann, der ein Buch liest. "Praeteritum, bedeutet/ das/ so gantz geschehen oder getahn ist/ wie der Dieb/ dem die Hände gebunden sind/ der hat gestolen" ([Olearius] 1630: 64). 


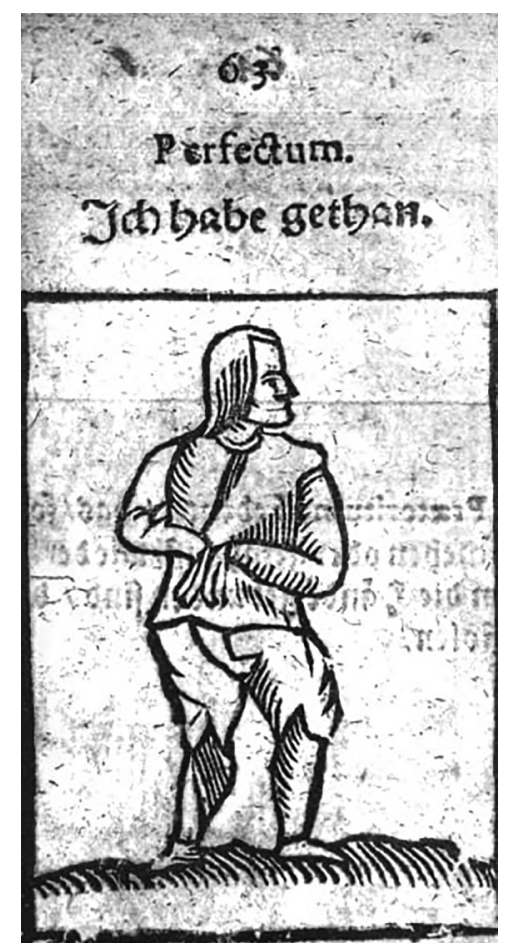

Abbildung 6. Das Praeteritum perfectum (1630); Quelle: ([Olearius] 1630: 63)

Hier handelte es sich um keine Figur eines Mannes, der als Personifikation der Zeit ausgewiesen war, sondern nur um den über Gefühle informierenden Gesichtsausdruck, sowie um die über die Intensität Auskunft gebende Gestik und Körperhaltung. Wie Panofsky schreibt:

Im Fall einer vorikonographischen Beschreibung, die sich im Rahmen der Motivwelt hält, scheint die Angelegenheit recht einfach zu sein. Die Objekte und Ereignisse, deren Darstellung durch Linien, Farben und Volumen die Motivwelt bilden, lassen sich, wie wir gesehen haben, auf der Grundlage unserer praktischen Erfahrungen identifizieren. Jedermann kann die Gestalt und das Verhalten menschlicher Wesen, von Tieren und Pflanzen erkennen, und jedermann kann ein zorniges Gesicht von einem fröhlichen unterscheiden. Natürlich ist es möglich, dass in einem bestimmten Fall das Spektrum unserer persönlichen Erfahrungen nicht umfassend genug ist, so etwa, wenn wir uns der Darstellung eines veralteten oder unvertrauten Werkzeugs oder der Darstellung einer Pflanze oder eines Tieres gegenübersehen, die uns nicht bekannt ist. In solchen Fällen müssen wir das Spektrum unserer praktischen Erfahrungen dadurch erweitern, dass wir ein Buch oder einen Fachmann befragen; doch wir verlassen nicht den Bereich praktischer Erfahrungen als solche, die uns - selbstredend - sagen, welcher Fachmann zu befragen ist (Panofsky 1975: 43).

Aufgrund persönlicher Erfahrungen sind die Schüler in der Lage, mimische Merkmale zu erkennen, wie zum Beispiel Schmerz, Trauer, Freude, Zorn, Mitleid, und die Bedeutung von bestimmten Gesten (Körpersprache) zu deuten. 


\section{Die Verwendung der Abbildungen des menschlichen Körpers in den deutschen und niederländischen Grammatiken des Barock in Abhängigkeit von den sprachwissenschaftlichen und religiösen Ansichten der jeweiligen Autoren}

Seit jeher lassen sich zwei unterschiedliche Herangehensweisen an die Geschichte der Sprachwissenschaft ausmachen: ein logisch-onomasiologischer und ein formal-semiologischer Zugriff. Als besonders prägend für die Entwicklung des humanistischen Bildungsdenkens wirkte sich der Einfluss Philipp Melanchthons (1497-1560) aus. Seine lateinische Grammatik war das wichtigste Lateinlehrbuch im 16. und 17. Jahrhundert. Insgesamt dominiert bei Melanchthons Definition der Kategorien eindeutig die deduktive Verfahrensweise. Seine Deduktion ist meistens auf die semantische Fragestellung eingeschränkt und geht auf die Gegebenheiten der Ontologie zurück, aus denen die sprachlichen Elemente abgeleitet werden. Er stellt die Semantik in den Vordergrund. Er greift auf die formalen Kriterien in der Regel erst zurück, wenn die semantischen ausgeschöpft sind:

Nomen est pars orationis, quae rem significat, non actionem ... ut Homo, Equus, Animal [...] Verbum est uox, significans agere aliquid aut pati, ut: Verberare actionem significat, Verberari passionem (Melanchthon 1561: 13, 201).

Melanchthon diente den deutschen Grammatikern als Vorbild für die logischsemantische Methode. Im deutschen Sprachraum herrschen diejenigen Grammatiken vor, die die semantisch-ontologischen Prinzipien vorziehen. In den Lehrbüchern der deutschen Sprache des 16. und des 17. Jh., z.B. in den Grammatiken von Laurentius Albertus, Johannes Clajus, Wolfgang Ratke und Christoph Helwig, sieht man den Übergang zur semantischen Behandlungsweise, indem der an der Semantik orientierte Ansatz Melanchthons weiter entwickelt wurde. Vom logisch-semantischen Standpunkt aus entsprechen die Sprachen strukturell der Realität und dem Denken. Bei einigen im 17. Jahrhundert erschienenen Universalgrammatiken wird der Bezug zur Realität, bei anderen der Bezug zum Denken stärker betont (Weiß 1992: 19).

Die obengenannte Grammatik des Olearius ist ein Beispiel für die Art einer universellen Grammatik. Die Benutzung der Bilder für die Erklärung der grammatischen Erscheinungen in Olearius' Werk ermöglicht das Erlernen verschiedener Sprachen und verleiht der Grammatik einen universellen Charakter. Der universelle Charakter der Grammatik des Olearius wird mit den Gesten noch mehr betont, denn die Bedeutung der Gesten eines Menschen ist über nationale Grenzen hinweg verständlich. Olearius spricht sich dafür in dem Appendix seiner Grammatik aus: 
Dergestalt / dann dieses Büchlein nicht allein ein Compendium der Deutschen Grammaticae, sondern es sind zugleich darin generalissima praecepta, in vielen andern Sprachen zugebrauchen ([Olearius] 1630: 89).

\section{George Arthur Padley schreibt dazu:}

For this little book is not merely a compendium of German grammar, but it also contains generalissima praecepta which can be used with many other languages (Padley 1985: 119).

Und bei Arnd Friedrich lesen wir:

Nach der Mitte des 16. Jahrhunderts war die absolute Vorrangstellung Melanchthons in Deutschland, soweit es seine pädagogischen Absichten betraf, zwar erschüttert, aber keineswegs gebrochen. Neben den Melanchthonianismus trat der Ramismus (Friedrich 2000, II: 717).

\section{Über Ramus' sprachtheoretische Haltung schreibt Erika Ising:}

In scharfem Gegensatz zur ontologisch gerichteten Grammatik des Spätmittelalters, deren sprachliche Analyse vor allem dem Wort als Teil eines geformten Satz- und Bedeutungszusammenhanges galt, hatte Petrus Ramus die Grammatik auf die Feststellung der rein formal-logischen Funktionen der Sprache beschränkt. Bei ihrer Darstellung untersuchte er unter 'grundsätzlichem Negieren der semasiologischen Aspekte' lediglich die äußere Sprachform (Ising 1959: 40).

Es gab auch lateinische Grammatiken, die im deutschen Sprachraum verfasst wurden und die der semantisch orientierten Grammatiktradition nicht folgten. 1620 erschien das Compendium grammaticae latinae: Ad didacticam (Compendium 1620), dessen Verfasser vermutlich Nicolaus Pompeus, Mitarbeiter von Ratke, war. Das Compendium weicht nun von Ratkes Allgemeiner Sprachlehr (Ratke 1619) nicht nur in Einzelfragen, sondern bereits im Gesamtkonzept ab. Zunächst gibt diese Schrift nicht vor, eine Universalgrammatik zu sein. Sie hat zwar instrumentellen Charakter (und wird als habitus instrumentalis definiert), bezieht sich jedoch im Wesentlichen auf die Formbildung der lateinischen Sprache (als ein Habitus dictionum latinarum conjungendarum, ad latinitatem). Die Norm wird doppelt definiert, allerdings mit einem deutlichen Schwerpunkt auf die auctoritas: "Latinitas est congruentia sermonis secundum artem, cum probatis autoribus latinis" (Compendium 1620: 3; vgl. Kordes 2004: 486 f.). Diese lateinische Grammatik ist

als ramistisch zu bezeichnen. Damit unterscheidet sie sich vor allem [...] von den noch durch Ratke beeinflussten Köthener Universalgrammatiken. Wie bei Ramus wird die Bedeutung in dieser lateinischen Grammatik vollständig aus den Definitionen ausgeklammert, es gelten nur die innerhalb der Grammatik als Kategorien begründbaren Akzidenzien bzw. ihre Veränderungen (Kordes 2004: 486 f.; Djubo 2008: 93 f.).

Viele deutsche Verfasser der Sprachlehren gingen vom Glauben an die Effektivität ihrer didaktischen Methode beim Sprachunterricht aus. Die gesteigerte Zuwendung zu Methodenfragen war ein verbindendes Element. Dadurch wurde 
aber die theologische Fraktionsbildung bei Lutheranern und Calvinisten nicht aufgehoben. Das klare rationale Denken Calvins stand im Widerspruch zu Luthers gefühlsbetonter Denk- und Ausdrucksart.

Während Martin Luther und seine Anhänger solche Bilder, die mit den reformatorischen Glaubensinhalten konform waren, religiös legitimierten (da es im Alten Testament kein radikales und umfassendes Bilderverbot gibt), haben Zwingli und Calvin die Verehrung der Bilder als Götzendienst hingestellt und die Beseitigung der Bilder verlangt. Im Einflussbereich von Zwingli und Calvin sollten alle Bilder aus den Kirchen verschwinden (vgl. Ortag 2008: 74; Iserloh 1974: 75-85).

In seinem theologischen Hauptwerk für die reformierte Kirche Institutio Christianae religionis (Unterricht in der christlichen Religion) widmet Calvin den Bildern ein Kapitel mit dem Titel "Es ist Sünde, Gott sichtbare Gestalt beizulegen; völliger Abfall vom wahren Gott ist es, wenn man sich Götzenbilder macht" (Calvin 1997: 40). Luther dagegen erkannte sehr wohl, dass die bildende Kunst ein wirksames Mittel sein konnte, den Menschen den christlichen Glauben zu vermitteln und erklärte: "eyn cruzifix oder heyligen bilde lasse zum ansehen, zum zeugnis, zum gedechtnis, zum zeychen" (Luther 1908: 80).

Das biblische Bilderverbot wird auf die Grammatiken der reformierten Verfasser extrapoliert. Einer der Grundzüge "der reformierten Ästhetik ist eine gewisse Schlichtheit oder Nüchternheit, die dem protestantischen Prinzip des Sola gratia in Verbindung gebracht werden können" (Stückelberger 2005: 17). Eine Gedächtniskunst, die auf eine bilderlose dialektische Ordnung als die wahre natürliche Ordnung des Geistes gegründet ist, passt gut zur calvinistischen Theologie (siehe Yates 2001: 219).

Durch Gliedern und Zusammenfassen des Stoffes sollten bei Ramus die emotional eindrucksvollen, das Gedächtnis stimulierenden Bilder durch eine der Vernunftnatur des Gedächtnisses entsprechende Ordnung abgelöst werden. Ramus, selbst Protestant, habe damit, so meint jedenfalls die englische Historikerin Frances Yates, den protestantischen Bildersturm durch eine Art "inneren Ikonoklasmus" fortgesetzt (siehe Rohl 2006: 12-13). Yates schreibt dazu:

Die Gestalt der alten Grammatica in einer Skulpturenreihe der freien Künste am Portal einer Kirche würde somit in einem Land, in dem der Protestantismus ungezügelt um sich griffe, äuBerlich auf dieselbe Art behandelt wie innerlich im Ramismus. Sie würde zertrümmert (Yates 2001: 217).

Die religiös-philosophische Lehre des Ramismus setzte sich vor allem in den reformierten Lehranstalten Deutschlands durch. Ramus' Anhänger leiteten aus seinen Arbeiten

ein Ideal stilistischer Schlichtheit und argumentativer Stringenz ab, das sich im Norden und Osten Deutschlands gelegentlich mit einem strengen Protestantismus verband, dem von seinen Gegnern eine gewisse Nähe zum Calvinismus nachgesagt wurde (Gardt 1994: 268). 
Die Visualisierung mit Hilfe von Bildern des menschlichen Körpers hatte eine Veränderung des Wissens im Sinne einer Sensualisierung zur Folge. Statt dessen ist die Anschauungsdarstellung des Materials in Tabellen, die dessen konsequente Teilung in verschiedene Teilzahlen (u.a. Dichotomie) enthalten, in den Werken von Johann Thomas Freigius (1543-1583) vorhanden. Seit Ramus' Tod 1572 sah Freigius, Rektor des Altdorfer Gymnasiums, in sich seinen Nachfolger.

Freigius versuchte seit den frühen 1570-er Jahren die Anwendung der ramistischen Methode auf ein breites Spektrum der zeitgenössischen Wissenschaften (siehe Mährle 2000: 196). Die Verwendung der ramistischen Methode in Freigius' Publikationen in den frühen 1570er Jahren wies zwei Merkmale auf: Begriffe dichotomisch aufzuspalten und Dispositionen von Begriffen mit Hilfe von diagrammatischen Schautafeln zu visualisieren:

Freigius' Grammatiken waren im Vergleich zu den Studienbüchern Melanchthons von weit geringerem Umfang. [...] Um das Studium zu erleichtern, versuchte er, die grammatischen Regeln möglichst anschaulich zu vermitteln. Er gliederte deshalb den Lehrstoff mit Hilfe der dihairetischen Methode und fügte in seine Lehrbücher ramistische dichotomische Schemata ein (Mährle 2000: 237-238).

Der deutsche Pädagoge und Polyhistor Georg Andreas Fabricius (15891645), der in Oldendurg, Mühlhausen und Göttingen lebte, war Autor von enzyklopädischen Werken nach ramistischen Prinzipien (Jaumann 2004: 258). In seinem Thesaurus philosophicus sive Tabulae totius philosophiae systema behandelt er unter mnemotechnischen Aspekten 376 Tabellen für den Unterrichtsgebrauch, u.a. für die Grammatik (Fabricius 1624: 25-48).

Wenden wir uns in diesen Ausführungen nun der niederländischen Sprache zu. Diese war im Mittelalter noch im niederfränkischen Teil des Dialektkontinuums der deutschen Sprache, es gab aber im 17. Jahrhundert schon zwei ausgeprägte Schriftsprachen: das (Hoch-)Deutsche und das Niederländische. An der Ausbildung der niederländischen Schrift- und Literatursprache hatten die niederländischen Grammatiker zwischen 1550 und 1650 einen wichtigen Anteil (Weber 1987: 126).

Die erste in der Volkssprache geschriebene Grammatik erscheint für das Niederländische früher als für das Hochdeutsche. Es ist die Twe-spraack vande Nederduitsche letterkunst, höchstwahrscheinlich von Hendrik Laurenszoon Spieghel (1549-1612) verfasst und von der Amsterdamer Kamer in Liefde Bloeyende 1584 herausgegeben (Weber 1987: 127). Der Autor ist nicht mit Sicherheit festzustellen. Man vermutet, dass der Katholik Spieghel Mitverfasser war, und dass sich auch Dirck Volkertszoon Coornhert (1522-1590) und Roemer Visscher (1547-1620), beide Katholiken mit freieren Ansichten, an Spieghels grammatischen Untersuchungen beteiligten. Die Twe-spraack... enthält auch eine einige Jahre früher geschriebene Voorreden van de noodich en nutticheyt der Nederduytscher taelkunste, verfasst von Coornhert (Weber 1987: 127). Das Werk wurde zu Ehren 
der Bürgermeister von Amsterdam geschrieben. In ihrer Widmung verkünden die Amsterdamer Rederijkers, dass es ihnen darum geht, die Muttersprache zu erheben und zu verherrlichen.

Obwohl das Werk unter Einfluss des grenzüberschreitenden humanistischen Gedankengutes stand (die Verwendung lateinischer Formen auf dem Gebiet der Morphologie und der Syntax verlieh dem Niederländischen einen respektablen Charakter) und obwohl sich Spieghel konfessionell abgesondert hielt und katholisch blieb, fühlt man in seinem Werk einen Einfluss des strengen Protestantismus, dem die Schlichtheit und argumentative Stringenz eigen sind. In der Ausgabe der Twe-spraack... von 1614 gibt es nur eine Darstellung Christi am Anfang des Buches: auf dem Wappenschild der Amsterdamer Kamer ([Spieghel] 1614: A1).

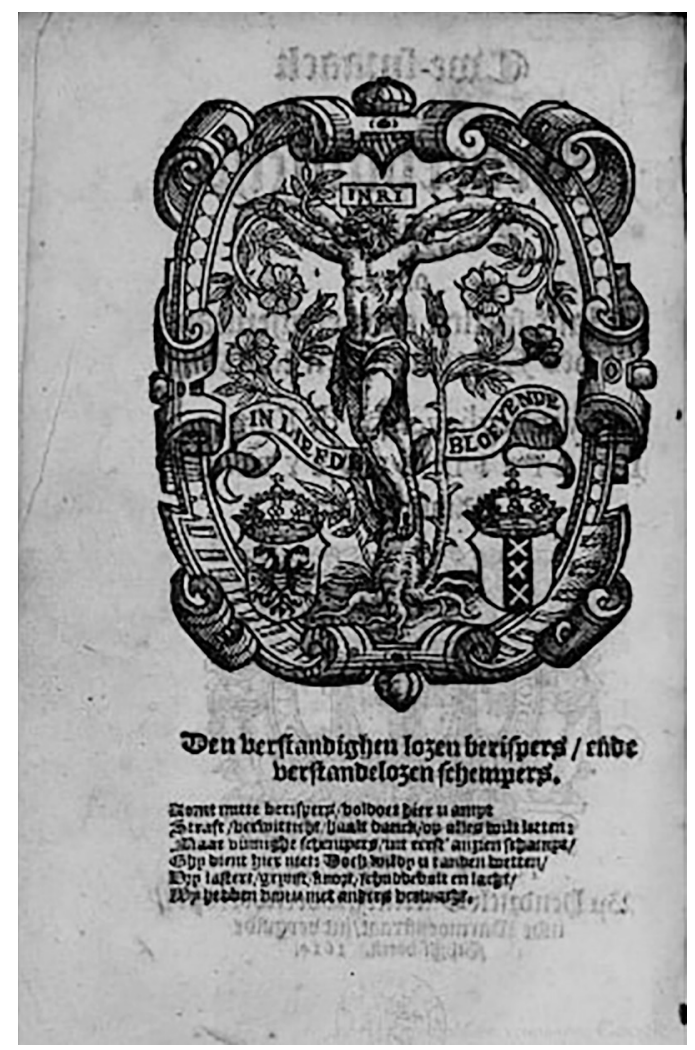

Abbildung 7. [Spieghel] 1614: A1v

Das Bild Christi mit der Inschrift INRI wiederholt sich in der 1614 vom Buchdrucker Hendrick Barentzs herausgegebenen Grammatik, die der Erstausgabe von 1584 im Druckhaus des frommen Katholiken Christoph Plantin folgte und nur eines der Markenzeichen des Druckers ist. Weitere Bilder fehlen im Buch. Es gibt aber ein paar Tabellen (vgl. ([Spieghel] 1614: 188, 238). 


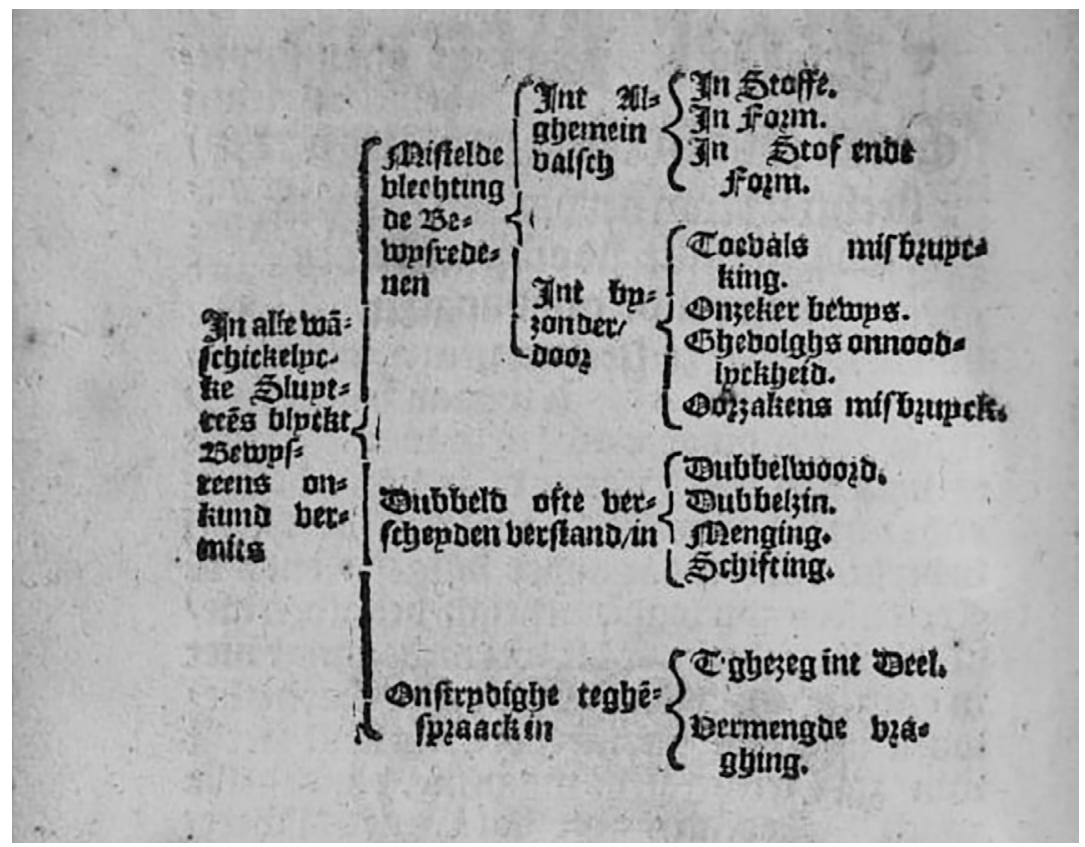

Abbildung 8. Tabelle aus [Spieghel] 1614: 238

Im Großen und Ganzen ließen die katholischen Grammatiker die Bilder in ihren Werken veröffentlichen. Dies war der Fall bei dem niederländischen Arzt, Linguist und Humanist Johannes Goropius Becanus [Jan van Gorp van der Beke] (1519-1572), der sich 1554 als Stadtarzt in Antwerpen niederließ. Seine letzten Jahre verbrachte er in Lüttich. Er liegt in der Franziskanerkirche von Maastricht begraben (über seine Zugehörigkeit zur katholischen Kirche siehe Schmidt 2007: 237).

Goropius war Verfasser von zwei berühmten Werken, Origines Antwerpianae (Antwerpen 1569) und Opera hactenus in lucem non edicta (Antwerpen 1580), in denen er zu beweisen versuchte, dass seine Muttersprache, also das Niederländische, die älteste in der Welt war [Ioan. Goropii Becani Origines Antwerpianae. Antverpiae: Plantinus, 1569: 460; Hispanica Ioannis Goropii Becani. Antverpiæ, Ex officina Christophori Plantini Architypographi Regij, 1580: 10] (siehe Kiedroń 1991: 10).

In seinem mehrteiligen Werk Opera Ioan[nis] Goropii Becani, hactenus in lucem non edita: nempe, Hermathena, Hieroglyphica, Vertumnus, Gallica, Francica, Hispanica (Goropius 1580) sind die Menschenbilder verschieden vertreten. Im Teil Gallica gibt es Darstellungen von Männerfiguren nur auf der Titelseite mit Plantins Motto "Labore et Constantia". Wir begegnen den Menschenkörpern auch in Initialen, d.h. in großen, künstlerisch verzierten Buchstaben am Anfang eines Kapitels. Im Teil Hieroglyphica gibt es viele Illustrationen, die die Geschichte der Buchstaben erzählen, z.B: 


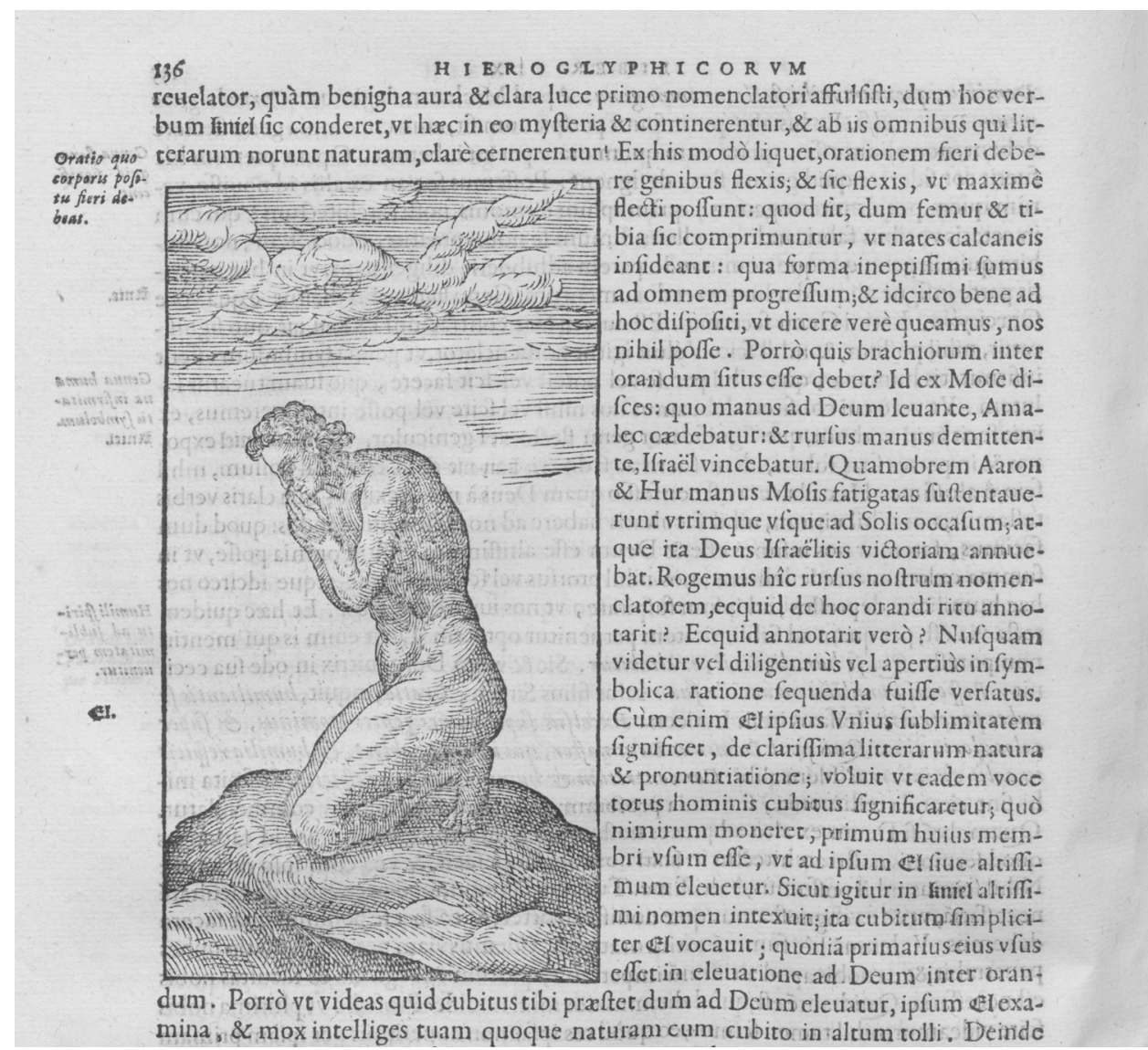

Abbildung 9. Goropius, 1580, Hieroglyphica (mit freundlicher Genehmigung der Abteilung für Alte Drucke der Biblioteka Uniwersytecka Wrocław); Quelle: Goropius 1680: 136

Die Opera... wurden noch vor der Eroberung Antwerpens 1585 durch die Spanier veröffentlicht, als viele Protestanten diese Stadt verließen. Dem Herausgeber gelang es jedoch bereits 1580, das Werk des 1572 verstorbenen katholischen Verfassers zu veröffentlichen, weil er schon am 16. November 1574 eine Druckerlaubnis vom Censor des apostolischen Sitzes Johannes Molanus bekommen hatte:

Joh. Goropij, piae memoriae, Hispanica, Francica, Gallica, Vertumnus, Hermathena, \& Hieroglyphica, eusdem sunt eruditionis cum iis operibus quae viuus edidit. Nec quicquam in his libris à me, aut ab aliis est aniamduersum quod fidem aut morum integritatem laedat. 16. Nouemb. 74. Ioh. Molanus, Apostolicus \& Regius censor" (Goropius 1580, Hispanica: 118).

Als Beispiel einer schlichten, vom Geist calvinistisch-reformierter Nüchternheit geprägten, relativ emotionslos verfassten Grammatik kann das Werk des niederländischen, aus calvinistischer Familie stammenden Gelehrten, Humanisten 
und Theologen Gerhard Johannes Vossius (1577-1649) dienen. Die lateinische Schulgrammatik des Vossius Latina grammatica wurde 1626 im Auftrag der nordniederländischen Generalstaaten in Leiden veröffentlicht. In der Einführung wird darüber folgendes geschrieben:

Id cum illustres Hollandiae \& VVest-Frisiae D.D. Ordines indignum plane, nes ulterius ferendum iudicarent: decretum fecere huiusmodi, ut eadem Latina lingua praecepta per omnem obtinerent provinciam; atque ut in adornanda eius editione nova, cuius mibi onus iniunctum fuit, ratio inprimis haberentur Ludolffi Lithocomi sed ita haberetur, ut, \& veritas in praeceptis.

Diese Grammatik war eine Zusammenstellung der Ideen seiner Vorgänger, die hier kritisiert wurden. Der Verfasser befolgt die philippo-ramistische Tradition in der Grammatikographie der ersten Hälfte des 17. Jahrhunderts, indem er rationalistische und empirische Vorgehensweisen in der Grammatikschreibung verwendet (vgl. die Definition des Verbs, in der die formalen und semantischen Kennzeichen dieses Redeteils genannt werden: "Verbum est dictio variabilis, quae agere, pati, vel esse significat"; Vossius 1626: 58).

In dieser, wie auch in den späteren Ausgaben, wie z.B. in der dritten Auflage (Vossius 1631), fehlen Bilder und Tabellen. Es gibt nur ein Verlagszeichen auf dem Titelblatt, das einen Baum und einen Menschen darstellt. Auf Verlangen von Schulrektoren, Universitätslehrern und für das Erziehungswesen zuständigen Beamten ordnete und ergänzte Vossius diese Notizen, so dass ein umfangreiches Werk systematischen Gepräges entstand: De Arte Grammatica Libri Septem (Vossius 1635), wo die Bilder und Tabellen ebenfalls fehlen.

Aus seinem Nachlass wurde noch ein gewichtiges Werk zur lateinischen Sprachwissenschaft in Amsterdam herausgegeben: Etymologicon linguae Latinae (Vossius 1662). Der calvinistischen Tradition entsprechend fehlen auch hier Bilder. Die Erlaubnis zum Veröffentlichen des Buches wurde vom Kaiser des Heiligen Römischen Reiches Leopold I. (1640-1705) gegeben. Auf Befehl seiner Heiligen Kaiserlichen Majestät (Ad mandatum Sacrae Cesariae Maiestatis proprium) unterschrieben Wilderich von Walderdorff (1617-1680, 1660-1669 Reichsvizekanzler) und Johann Walderode von Eckhausen (1593-1674, 1630 Sekretär der lateinischen Expedition der Reichskanzlei, auch Protokollführer des Geheimen Rats und Sekretär des Reichshofrat, 1637 Reichshofrat) diese Erlaubnis am 3. März 1662. Das Buch hatte am 13. März 1662 auch das Privileg von den Abgeordneten der Staaten von Holland und West-Friesland bekommen, von denen sich die meisten zur calvinistischen Konfession bekannten. Unterschrieben war diese Erlaubnis von Johan de Witt (1625-1672), dem Ratspensionär von Holland und West-Friesland, sowie von Herbert van Beaumont (1607-1679), dem Staatssekretär dieser Provinz (Vossius 1662: *3v).

Die niederländischen protestantischen Grammatiker befolgen am stärksten die ramistische Tradition: es fehlen Bilder, es gibt aber Tafeln und die dichoto- 
mische Methode, die sich in der Regel in der Komposition der Grammatik und bei der Beschreibung der Kennzeichen der Redeteile beobachten lässt.

Christiaen van Heule (?-1655) war ein niederländischer Grammatiker in Leiden, wo er in der protestantischen Kirche St. Peter begraben wurde. Seine grammatischen Werke sind: De Nederduytsche Grammatica ofte Spraec-konst (1625) und De Nederduytsche spraec-konst ofte tael-beschrijvinghe (1633).

Die beiden Ausgaben unterscheiden sich vor allem in der „Etymologie“, die mehr als die Hälfte der jeweiligen Werke umfasst. 1625 hatte sich van Heule an die lateinische Grammatik des Lithocimus angeschlossen; 1633 orientiert er sich an Petrus Ramus und seiner Zweiteilung der Grammatik (Weber 1987: 129). Wie Ramus gebraucht auch er in seiner De Nederduytsche spraec-konst Tabellen (Heule 1633: $+15,+68,+80$ ), so z.B. bei der Unterteilung der Nomina:

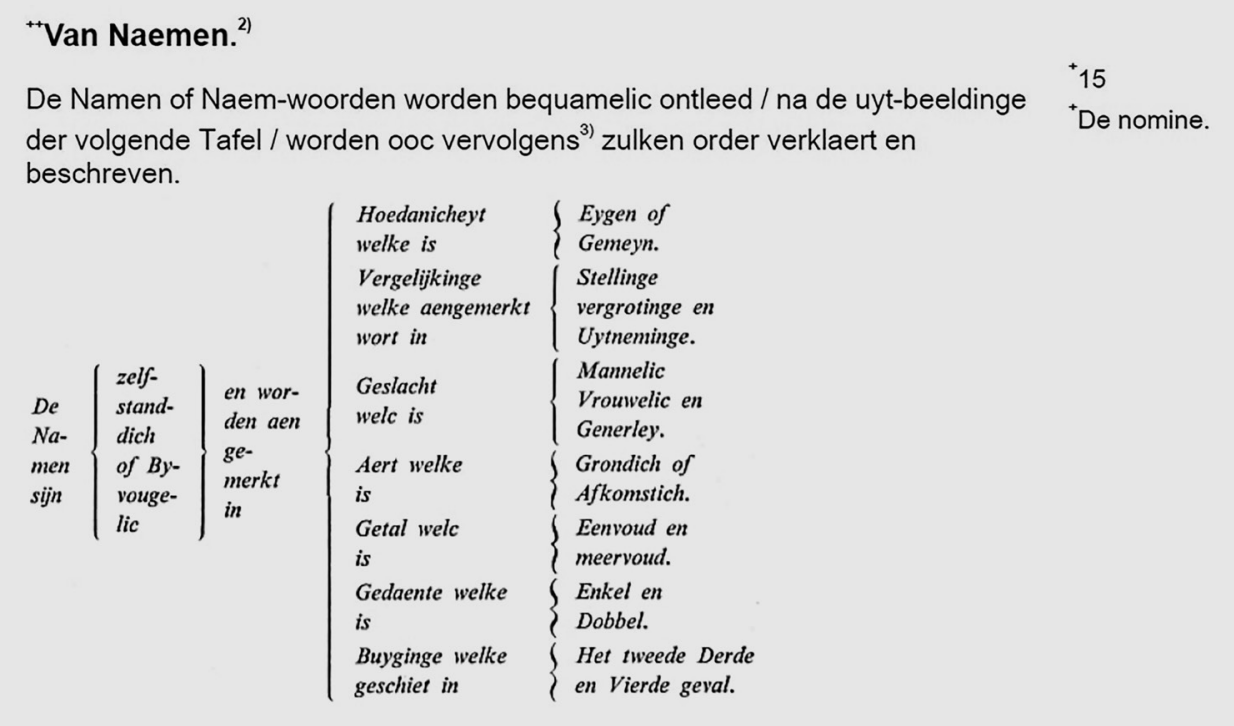

Abbildung 10. Tabelle Die Unterteilung der Nomina; Quelle: Heule 1633: +15

\section{Zusammenfassung}

Basierend auf den Meinungen von Forschern sowie auf der hier durchgeführten Analyse der anthropomorphen Bilder in den Grammatiken aus dem 16. und 17. Jahrhundert kann geschlossen werden, dass menschliche Figuren ein geeignetes Mittel waren, um die menschlichen Qualitäten den nicht-menschlichen Entitäten zuzuordnen und das abstrakte Phänomen dadurch konkreter und leichter verständlich zu machen. Die Grammatik wurde von personifizierten Frauen mit verschiedenen Kombinationen von Eigenschaften ausgestattet. Schon die ersten 
deutschen elementaren Grammatikwerke des 16. Jh. setzten zur Verbreitung des grammatischen Wissens zu den verbalen Regeln zusätzlich auf Visualisierungen. Die innerhalb des Textinnern und mit Bezug zu diesem eingesetzten Bilder enthalten keine Personifikation abstrakter Vorstellung, z.B. der grammatischen Kategorien.

Bei den Grammatikern, die wie Melanchthon eine Neigung zum Universalismus hatten, wurde die Visualisierung mit Hilfe von Bildern des menschlichen Körpers wie immer verwendet, weil die Verfasser damit eigene Ansichten und Gedanken belegten oder unterstützten.

Die Illustrierung oder nachträgliche Bebilderung von Grammatikwerken ist besonders in den elementaren Lehrbüchern verbreitet. Es hat aber solche gegeben, in denen die Bebilderung aus den grammatiktheoretischen und religiös-philosophischen Gründen ausgeschlossen war.

Nach der Mitte des 16. Jh. trat neben dem 'Melanchthonianismus' der 'Ramismus' auf. Die religiös-philosophische Lehre des 'Ramismus' setzte sich vor allem in den reformierten Lehranstalten Deutschlands und in den Niederlanden durch. Peter Ramus hat die Grammatik auf die Feststellung der rein formal-logischen Funktionen der Sprache beschränkt.

Viele Verfasser von Sprachlehren gingen vom Glauben der Effektivität ihrer didaktischen Methode beim Sprachunterricht aus. Die gesteigerte Zuwendung zu Methodenfragen war ein verbindendes Element, dadurch wurde die theologische Fraktionsbildung bei Lutheranern und Calvinisten aber nicht aufgehoben. Das klare rationale Denken Calvins stand zu Luthers gefühlsbetonter Denk- und Ausdrucksart im Widerspruch.

Eine Gedächtniskunst, die auf eine bilderlose dialektische Ordnung als die wahre natürliche Ordnung des Geistes gegründet ist, passt gut zur calvinistischen Theologie. Durch Gliedern und Zusammenfassen des Stoffes sollten bei Ramus die emotional eindrucksvollen, das Gedächtnis stimulierenden Bilder durch eine der Vernunftnatur des Gedächtnisses entsprechende Ordnung abgelöst werden. Die Visualisierung mit Hilfe von Bildern des menschlichen Körpers hat eine Veränderung des Wissens im Sinne einer Sensualisierung zur Folge. Die Anschauungsdarstellung des Materials in Tabellen, die dessen konsequente Teilung in verschiedene Teilenzahl (u.a. Dichotomie) enthalten, ist dagegen in den Werken jener deutschen und niederländischen Grammatiker vorhanden, die seit Ramus' Tod 1572 sich als seine Nachfolger sahen.

\section{Bibliographie}

Calvin, Jean (1997): Unterricht in der christlichen Religion = Institutio Christianae religionis. Nach der letzten Ausgabe (1559) übersetzt und bearbeitet von Otto Weber. Neukirchen-Vluyn: Neukirchener Verlag, 6. Auflage der einbändigen Ausgabe. 
Compendium grammaticae latinae: Ad didacticam (1620): Cothenis Anhaltinorum.

Djubo, Boris (2008): "Tradition und Wandlungsprozesse in der Grammatikographie der ersten Hälfte des 17. Jahrhunderts: zu Christian Gueintz' Grammatik". Wolfenbütteler Barock-Nachrichten 35:2, 93-114.

Fabricius, Georg Andreas (1624): Thesaurus philosophicus sive Tabulae totius philosophiae syste$m a$. Brunsvigae: Duncker.

Fijałkowski, Adam (2008): "Die 'voces variae animantium' in der Unterrichtstradition des Mittelalters und der Frühen Neuzeit". In: Andreas Speer \& David Wirmer (Hrsg.), Das Sein der Dauer. Berlin u.a.: De Gruyter, 447-472 (= Kölner Mediaevistentagung, 35).

Firth, Raymond (1990): “Jednostkowe symbole i publiczne reakcje”. In: Michał Głowiński (Hrsg.), Symbole i symbolika. Warszawa: Czytelnik, 241 (übers. Ignacy Sieradzki).

Friedrich, Arnd (2000): Das Pädagogium der Universität Marburg zwischen Melanchthonianismus und Ramismus. Melanchthon und die Marburger Professoren (1527-1627). Bd. 2. Marburg: Völker \& Ritter, 707-736.

Gardt, Andreas (1994): Sprachreflexion in Barock und Frühaufklärung. Berlin/New York: De Gruyter.

Glück, Helmut (2002): Deutsch als Fremdsprache in Europa vom Mittelalter bis zur Barockzeit. Berlin u.a.: De Gruyter.

Goropius, Johannes (1569): Origines Antwerpianae. Antverpiae: Plantinus.

Goropius, Johannes (1680): Opera Ioan[nis] Goropii Becani, hactenus in lucem non edita: nempe, Hermathena, Hieroglyphica, Vertumnus, Gallica, Francica, Hispanica. Antverpiae: Christoph Plantin.

Grüßbeutel, Jacob (1534): Eyn Besonder fast nützlich stymmen büchlein mit figuren, welche die stymmen an jn selbs anzeygen, mit silben vnd namen. In welchem die Gesellen, Eehalten, ṽ̃ ander alt leut, auch die kinder, weib vnd mañ, bald (als in XXIIII stunden auff das minst) leychtlich mögen lernen lesen: Mit eynem gegründten fast schönen Benedicte vnd Gratias zum Tisch die jungen kinder zu lernen. Augspurg: Gutknecht.

Grüßbeutel, Jacob (1882). Eine Frau, die Hühner vor sich hertreibt aus Eyn Besonder fast nützlich stymmen büchlein mit figuren. Augspurg: Gutknecht 1534. In: Heinrich Fechner (Hrsg.), Vier seltene Schriften des sechzehnten Jahrhunderts. Berlin: Wiegandt und Grieben 1882. Nr. 4. S.B.

Hanns, Robert (1881): "Beiträge zur Geschichte des Deutsch-sprachlichen Unterrichtes im siebzehnten Jahrhundert". Jahrbücher für Philologie und Paedagogik. Zweite Abteilung, 1-38, 65-87.

Hombach, Klaus Sachs (2002): "Bildbegriff und Bildwissenschaft”. Kunst-gestaltung - design 8, $3-26$.

Iserloh, Ervin (1974): "Die Verteidigung der Bilder durch Johannes Eck zu Beginn des reformatorischen Bildersturms". Aus Reformation und Gegenreformation. Festschrift für Theobald Freudenberger, 75-85 (= Würzburger Diözesan-Geschichtsblätter, 35-36).

Ising, Erika (1959): Wolfgang Ratkes Schriften zur deutschen Grammatik (1612-1630). Bd. 1. Abhandlung, Bd. 2. Textausgabe. Berlin: Akad.-Verl.

Jaumann, Herbert (2004): "Ramus, Petrus". In: Handbuch Gelehrtenkultur der frühen Neuzeit. Bd. 1. Bio-bibliographisches Repertorium. Berlin u.a.: De Gruyter, 541-543.

Jordan, Peter (1533): Leyenschul. Gedruckt zu Meyntz.

Jordan, Peter (1882): Von den halblauten buchstaben aus Leyenschul. Gedruckt zu Meyntz 1533. In: Heinrich Fechner (Hrsg.), Vier seltene Schriften des sechzehnten Jahrhunderts. Berlin: Wiegandt und Grieben. Nr. 3. S. Avj.

Kiedroń Stefan (1991): Niederländische Einflüsse auf die Sprachtheorie von Justus Georg Schottelius. Wrocław: Wydawnictwo Uniwersytetu Wrocławskiego.

Kordes, Uwe (2004): "Wissenschaftstheorie, Grammatik, Didaktik. Wolfgang Ratke (Ratichius; 15711635)". In: History of Linguistics in Texts and Concepts. Bd. II, 479-492.

Luther, Martin (1908): Martin Luthers Werke: kritische Gesamtausgabe. Bd. 18. Weimar: Böhlau.

Neerlandica Wratislaviensia 29, 2019

(C) for this edition by CNS 
Mährle, Wolfgang (2000): Academia Norica: Wissenschaft und Bildung an der Nürnberger Hohen Schule in Altdorf (1575-1623). Stuttgart: Franz Steiner.

Melanchthon, Philipp (1561): Grammatica Latina. Lipsiae: Papa.

Mittelberg, Irene (2002): "The visual memory of grammar: Iconographical and metaphorical insights". Metaphorik.de (The Visual Memory of Grammar), 69-89.

Moulin, Claudine (2008): "Grammatik im Bild-Bilder der Grammatik: Räume des sprachlichen Wissens in der Grammatikographie der Frühen Neuzeit”. In: Gewusst wo! - Wissenschafft Räume: die Verortung des Denkens im Spiegel der Druckgraphik (Ausstellung im Gutenberg-Museum, 31.10.2008 bis 29.03.2009). Berlin: Akad.-Verl., 17-36.

Moulin, Claudine (2011): "Grammatische Architekturen. Zur Konstitution von metasprachlichem Wissens in der Frühen Neuzeit”. In: Sabine Frommel \& Gernot Kamecke (Hrsg.), Les sciences humaines et leurs langages. Artifices et adotions. Roma: Campisano, 25-44.

Müller, Johannes (1882): Quellenschriften und Geschichte des deutschsprachlichen Unterrichtes bis zur Mitte des 16. Jahrhunderts. Gotha: Thienemann.

Nasalski, Ignacy (2004): Die politische Metapher im Arabischen: Untersuchungen zu Semiotik und Symbolik der politischen Sprache am Beispiel Ägyptens. Wiesbaden: Harrasowitz.

[Olearius, Tilemann] (1630): Deutsche Sprachkunst: Aus den allergewissesten/der Vernunfft un [d] gemeinen brauch Deutsch zu reden gemässen/gründen genommen; Sampt angehengten newen methodo, die Lateinische Sprache ... zu lernen/. Hall: Oelschlegel.

Ortag, Peter (2008): Christliche Kultur und Geschichte: ein Überblick. Potsdam: Brandenburgische Landeszentrale $\mathrm{f}$. politische Bildung.

Padley, George Arthur (1985): Grammatical Theory in Western Europe, 1500-1700: Trends in Vernacular Grammar. Bd. 1. Cambridge u.a.: Cambridge University Press.

Panofsky, Erwin (1975): Sinn und Deutung in der bildenden Kunst (Meaning in the Visual Arts) (1955). Köln: DuMont Schauberg (übers. Wilhelm Höck).

Ratke, Wolfgang (1959): “Allgemeine Sprachlehr: Nach der Lehrart Ratichii”. In: Erika Ising (Hrsg.), Wolfgang Ratkes Schriften zur deutschen Grammatik (1612-1630). Teil 2. Berlin: AkademieVerlag, 23-37, 38-48.

Rohl, Klaus F. (2006): Die Bedeutung der Schrift für die Bildabstinenz der Juristen. Bausteine für das Projekt 'Visuelle Rechtskommunikation'. Ruhr-Universität Bochum. Lehrstuhl für Rechtssoziologie und Rechtsphilosophie, zuletzt konsultiert am 26.03.2006 <http://www.rechtssoziologie.info/literatur/texte/projekt-visuelle-rechtskommunikation/schrift_und_bild.pdf $>$.

Schmidt, Alexander (2007): Vaterlandsliebe und Religionskonflikt: politische Diskurse im Alten Reich (1555-1648). Leiden u.a.: Brill.

Schnettler, Bernt, \& Frederik S. Pötzsch (2007): "Visuelles Wissen”. In: Rainer Schützeichel (Hrsg.), Handbuch Wissenssoziologie und Wissensforschung. Konstanz: UVK Verlagsgesellschaft mbH., 472-484.

Schottel, Justus Georg (1663): Ausführliche Arbeit Von der Teutschen HaubtSprache. Braunschweig: Zilliger.

Spieghel, Hendrik Laurenszoon (1584): Twe-spraack vande nederduitsche letterkunst: ofte vant spellen ende eygenscap des nederduitschen taals. Leyden: Plantyn.

[Spieghel, Hendrik Laurensz] (1614): Twe-spraack vande Nederduitsche letterkunst, ofte, Vant spellen ende eyghenscap des Nederduitschen taals. Tot Amstelredam: by Hendrick Barentz.

Stückelberger, Johannes (2005): "Das unsichtbare Bild - Prolegomena zu einer reformierten Ästhetik". In: Matthias Krieg, Martin Rüsch, Johannes Stückelberger \& Matthias Zeindler (Hrsg.), Das unsichtbare Bild. Die Ästhetik des Bilderverbotes (Katalog der Ausstellung in den Kirchen von Zürich-Predigern, Kappel am Albis, Schaffhausen-Münster und Oberwinterthur, 30. Mai bis 15. Juli 2005). Zürich: Theologischer Verlag Zürich, 11-19.

Van Heule, Christiaen (1625): De Nederduytsche Grammatica ofte Spraec-konst. Leiden: Daniel Roels.

Neerlandica Wratislaviensia 29, 2019

(C) for this edition by CNS 
Van Heule, Christiaen (1633): De Nederduytsche spraec-konst ofte tael-beschrijvinghe. De tweede Druc verbetert. Tot Leyden: by Jacob Koels, zit. nach Christiaan van Heule (1953): De Nederduytsche spraec-konst ofte tael-beschrijvinghe, W.J.H. Caron (Hrsg.). Groningen/Djakarta: J.B. Wolters.

Vogtherr, Heinrich (1548): Der Turm der Grammatik (Holzschnitt). In: Max Geisberg (Hrsg.), The German Single-Leaf Woodcut: 1500-1550. New York: Hacker 1974, No. 1430.

Vossius, Gerhard Johannes (1626): Latina grammatica, In usum Scholarum Hollandiae, \& West-Frisiae... Ex Officina Bonaventvrae \& Abrahami Elzevir. Academiae Typograph.

Vossius, Gerhard Johannes (1635): De arte Grammatica Libri septem. Amsterdami: Apud Gvilielmvm Blaev.

Vossius, Gerhard Johannes (1662): Etymologicon linguae latinae. Praefigitur Ejusdem de literarum Permutatione tractatus. Amstelodami: Apud Ludovicum \& Danielem Elzevirios.

Vossius, Gerhard Johannes (1631): Latina grammatica, Ex decreto Illustr. DD. Holl. West-Frisiaeqae, in usum scholarum adornata... Lugduni Batavorum: Bonaventurae et Elzevir.

Weber, Heinrich (1987): "Die Ausbildung der deutschen Grammatik (einschliesslich der niederländischen)". Histoire Épistémologie Langage 9:1, 111-133.

Weiß, Helmut (1992): Universalgrammatiken aus der ersten Hälfte des 18. Jahrhunderts in Deutschland: eine historisch-systematische Untersuchung. Münster: Nodus-Publ.

Yates, Frances A. (2001): Gedächtnis und Erinnern. Mnemonik von Aristoteles bis Shakespeare, Berlin: Akademie Verlag. 